\title{
How asbestos and other fibers cause mesothelioma
}

\author{
Giovanni Gaudino, Jiaming Xue, Haining Yang \\ University of Hawai'i Cancer Center, Honolulu, HI 96813, USA \\ Contributions: (I) Conception and design: G Gaudino, H Yang; (II) Administrative support: H Yang; (III) Provision of study materials or patients: \\ G Gaudino; (IV) Collection and assembly of data: G Gaudino, H Yang; (V) Data analysis and interpretation: G Gaudino, H Yang; (VI) Manuscript \\ writing: All authors; (VII) Final approval of manuscript: All authors. \\ Correspondence to: Dr. Haining Yang. University of Hawai'i Cancer Center, 701 Ilalo street, Honolulu, HI 96813, USA. Email: hyang@cc.hawaii.edu.
}

\begin{abstract}
Mesothelioma has long been associated with the exposure to asbestos, which was largely used in manufacturing activities. Toxicology studies in vitro and in vivo demonstrated that asbestos fibers were carcinogenic, and epidemiology studies revealed that asbestos exposure was paralleled by the increase in the incidence of mesothelioma and related mortality rates. More recently, the role of chronic inflammation and the molecular mechanisms involved in carcinogenesis by mineral fibers were elucidated following the discovery of the roles of HMGB1 and inflammasome. A change of paradigm was the discovery of a prevalence of mesotheliomas attributable to inherited mutations of cancer susceptibility genes. The discovery of $B A P 1$ as a predisposition gene for the development of familial mesothelioma and other cancers implemented genome studies in patients with mesothelioma and routine clinical surveys in individuals at risk to identify germline mutations associated with cancers included in the BAP1 syndrome. A further progress in the approach to asbestos-related malignancy was the adoption of combined genetics and environmental analyses according to the model of gene-environment $(\mathrm{GxE})$ interactions. This review aims at updating on the most recently discovered mechanisms of tumorigenesis and the pivotal role of $\mathrm{GxE}$ interactions.
\end{abstract}

Keywords: Mesothelioma; asbestos; high mobility group box 1 (HMGB1); inflammasome; BRCA1 associated protein-1 (BAP1)

Submitted Jan 09, 2020. Accepted for publication Jan 30, 2020.

doi: $10.21037 /$ tlcr.2020.02.01

View this article at: http://dx.doi.org/10.21037/tlcr.2020.02.01

\section{Introduction}

Mesothelioma is a cancer arising from the transformation of mesothelial cells lining the thoracic (pleura) or the abdominal (peritoneum) cavities, and more rarely from transformed mesothelial cells of pericardium or tunica vaginalis testis. The development of mesothelioma has been related to the exposure to carcinogenic mineral fibers, mainly asbestos (1). The large and extensive utilization of asbestos since the mid- $20^{\text {th }}$ century, because of its insulating properties and low cost-effectiveness, was followed by a substantial increase in the age-standardized incidence of mesothelioma and related mortality rates until the developed countries strictly regulated (United States) or banned (Europe, Australia) the use of this material, after toxicological studies in vitro and in rodents demonstrated that asbestos fibers were carcinogenic (2).

The national regulatory documents use the generic term asbestos referring to six minerals that were commercially exploited, five amphiboles (crocidolite, actinolite, tremolite, anthophyllite, and amosite) and one serpentine (chrysotile). However, in the natural environment approximately further 400 minerals with similar physical and chemical features remain there, have not been regulated, and can be used unrestrictedly. The fibers of at least some of these minerals have been shown carcinogenic but can be airborne dispersed and affect the local human communities, drawing attention to the inadequacy of the current terminology (3). As an example, the residents of some Cappadocian villages in Turkey and in North Dakota (US) are exposed to naturally occurring erionite fibers, which are more carcinogenic than those of regulated asbestos, but have been used as 
construction or road paving materials $(4,5)$.

We review here the current mechanisms of mesothelioma tumorigenesis, focusing on the impact of carcinogenic mineral fibers on mesothelial cells, the related molecular responses, and the relevance of gene-environment $(\mathrm{GxE})$ interactions.

\section{Physicochemical features of the fibers govern their carcinogenic potential}

It has been shown by long time that dimensions, durability, and dose ("three D's") and physical properties are critical to determine the carcinogenic potency of certain types of mineral fibers (6-8). Fiber dimensions are related to durability and dose because influence bioavailability after inhalation. Long and thin fibers are associated with a higher potency regarding cytotoxicity and mutagenesis. A metaanalysis found that individuals exposed to fibers longer than $10 \mu \mathrm{m}$ and even $20 \mu \mathrm{m}$ have a significantly higher risk for asbestos-related disease (9), because longer fibers cannot be efficiently engulfed and cleared by macrophages leading to repeated failed attempts of phagocytosis. The resulting "frustrated phagocytosis" induces the inflammatory cells surrounding the fibers to release free radicals like reactive oxygen species (ROS) and reactive nitrogen species (RNS), exerting mutagenic activity $(7,10)$. According to the WHO (World Health Organization) asbestos fibers can be operationally distinguished in short asbestos fibers (SAF) with length $<5 \mu \mathrm{m}$ and long asbestos fibers (LAF), having length $>5 \mu \mathrm{m}$, diameter $<3 \mu \mathrm{m}$ and length/diameter ratio $>3$, which are targeted by the current regulatory rules (11). Moreover, differences in fiber biopersistence after exposure influence tumorigenesis, as serpentine chrysotile characterized by shorter biopersistence (12) compared with amphiboles and erionite, displays a lower carcinogenic potential. However, when the exposure to chrysotile fibers is prolonged, mesothelial cells are equally transformed (13). On the other hand, palygorskite (a very abundant fiber in southern Nevada) with reduced cytotoxicity and biopersistence in vitro, failed to induce carcinogenesis in vivo because it elicits much reduced inflammation compared to carcinogenic fibers (14).

\section{Fiber-related cell transformation}

The mechanisms of asbestos carcinogenesis have been elusive for long time and the initial hypothesis of the fiber mechanical interference with cell division has been definitely ruled out (2). However, some studies suggested that fiber chemical structure may play a role and iron in particular, as impurity or even as a chemical component of the fiber structure, has been taken into consideration to explain the carcinogenic process induced by asbestos. The deposition of asbestos fibers in tissues offers a surface where iron-reach macromolecular aggregates (asbestos bodies) favoring the development of chronic inflammation (15). Moreover, it has been shown that asbestos-activated macrophages release ROS that, in turn, may induce DNA damage indirectly via formation of 8-hydroxy-2'deoxyguanosine (8-OHdG) adducts (16). Recent data on iron-catalyzed ROS production suggests that ferroptosis, a non-apoptotic, iron-dependent cell death, may be involved in asbestos-related carcinogenesis (17). Moreover, a role in asbestos-induced carcinogenesis has been postulated for hepatocyte growth factor (HGF), via activation of the PI3K/MEK5/Fra-1 axis (18).

On the other hand, the exposure to asbestos fibers causes death of human mesothelial cell (HM), a cell type particularly susceptible to fiber cytotoxicity that was initially ascribed to apoptosis (19). Afterwards, asbestos pathogenesis was clearly associated with tumor necrosis factor-alpha (TNF- $\alpha$ ), a mediator of inflammation (20).

\section{Chronic inflammation and mesothelioma}

Chronic inflammation plays a major role in the pathogenesis and tumorigenesis induced by asbestos and other carcinogenic mineral fibers. The pro-inflammatory microenvironment established at the site of fiber deposition with the contribution of both HM and macrophages, combined with the biopersistence of many mineral fibers, allow some HM to avoid cell death and eventually go through oncogenic transformation (1).

It has been shown that the largest fraction of HM exposed to crocidolite (21) and chrysotile asbestos (13), as well as to erionite fibers (4), undergo cell death via programmed cell necrosis (21). This is a regulated form of necrosis characterized by the passive release of high mobility group box 1 (HMGB1) by necrotic HM at the site of fiber deposits. HMGB1 is a damage-associated molecular protein (DAMP) that promotes the recruitment of macrophages sustaining the chronic inflammatory process $(21,22)$. HMGB1 binds to RAGE and other HMGB1 receptors of macrophages priming for inflammasome activation, combined with other stimuli, like endogenous ROS formed after asbestos exposure, through the assembly 
of NLRP3 inflammasome via oligomerization of inactive NLRP3, apoptosis-associated speck-like protein (ASC) and procaspase-1. NLRP3 inflammasome induces the release of IL-1 $\beta$, IL-18, IL-1 $\alpha$, and HMGB1, establishing an autocrine chronic inflammation process (23). During this process also TNF- $\alpha$ is secreted and activates NF- $\kappa \mathrm{B}$, promoting survival of HM upon asbestos exposure. The surviving HM will continue to proliferate and accumulate genetic mutations, leading to development of mesothelioma (1). The role of HMGB1 and related chronic inflammation is supported by the report on the preventative role of aspirin for mesothelioma, targeting HMGB1 activities and inflammation, and on the antitumor activity of aspirin in mesothelioma xenograft models (24). Moreover, mesothelioma cell growth was inhibited both in vitro and in vivo by ethyl pyruvate that has been characterized as an effective inhibitor of HMGB1 and suppressor of the expression of the RAGE receptor. Both activities contribute to reducing mesothelioma malignancy (25).

The anti-tumor activity of these widely used antiinflammatory drugs is explained by the high levels of HMGB1 expression and secretion in the extracellular milieu found in mesothelioma cells, compared with HM and by the findings that competitive inhibitors of HMGB1 delay growth of mesothelioma xenografts (26). HMGB 1 is localized mainly in the nucleus of HM, while in mesothelioma HMGB1 was found in both nucleus and cytosol (26). The subcellular localization of HMGB1 is determined by the balance between histone acetyltransferase (HAT) and histone deacetylase (HDAC), controlling the HMGB1 acetylation status $(27,28)$, which is also regulated by poly(ADP-ribose) polymerase-1 (PARP-1) (29). In mesothelioma HMGB1 is actively secreted into the extracellular space (30), where it establishes an autocrine mechanism with RAGE and TLR receptors that promotes proliferation, motility, and survival, leading to the progression of mesothelioma (26).

\section{The role of genes and environment}

Asbestos fibers initiate HM death mainly through necrosis (21) and to a lesser extent by other cell death mechanisms (31), are also studied in parallel. Carcinogenesis is commonly related to somatic gene mutations affecting the DNA repair mechanisms, leading to the accumulation of DNA damage and the consequent increase of the fraction of cells carrying damaged DNA. When these cells acquire mechanisms of survival, as those elicited by the HMGB1 pathway in mesothelioma, cancer may develop. The presence of inherited mutations affecting DNA repair and other genes may further contribute to the process of carcinogenesis, by increasing the susceptibility to environmental carcinogens (32). The current approach adopted in the field of carcinogens is to combine genetics and environmental studies to study GxE interactions (2).

The catastrophic event of chromothripsis has been recently attributed to the increase of the mutational level of the cancer cell genome. Chromothripsis develops upon the shattering of a segregated single chromosome that is randomly reassembled, leading to incorrect rearrangements or deletions of DNA sequences. Therefore, even after a short number of cell divisions, massive genome alterations may occur following a single chromothripsis event. In turn, this high mutational status favors oncogene activations or loss of tumor suppressor functions, eventually promoting tumorigenesis (33). Notably, genomic studies of mesothelioma cells and specimens identified noncontiguous biallelic genome alterations with the distinctive pattern of chromothripsis $(34,35)$, and associated with potential neoantigen expression, with possible and intriguing implications in mesothelioma immunogenicity (36).

Multiple tumor suppressors involved in the cell cycle control and in apoptosis were found mutated in human mesothelioma. One of the common genetic alterations in mesothelioma is the homozygous deletion on locus 9p21 (37), which affects the transcription of two tumor suppressors: $\mathrm{p} 16^{\mathrm{INK} 4 \mathrm{a}}$ and $\mathrm{p} 14^{\mathrm{ARF}}$. P16 ${ }^{\mathrm{INK} 4_{\mathrm{a}}}$ blocks cell division via binding to CDK4 and CDK6, and p14 promotes apoptosis by inhibiting p53 ubiquitylation. Cytogenetic studies showed that p16 was missing in up to $80 \%$ primary pleural mesotheliomas (37), while p16 inactivation suggests the association with poor clinical outcome (38). Transgenic p14 (+/-) mice were more susceptible to asbestos-induced carcinogenesis and harvested primary mice tumors exhibited loss of heterogeneity for p14 (39).

Intermediates in the Hippo signaling pathway are also highly mutated in mesothelioma. Neurofibromatosis type 2 (NF2)/Merlin, an upstream initiator of Hippo, is inactivated in about $40 \%$ of malignant mesothelioma (40). Notably, NF2 is the second most frequent mutated gene in mesothelioma after BRCA1 associated protein-1 (BAP1). Heterozygous NF2 (+/-) mice were more sensible to asbestos exposure and demonstrated an accelerated tumorigenesis compared to wildtype controls (41). Nonfunctional NF2 leads to nuclear accumulation of yesassociated protein (YAP) and WW Domain-containing 
transcription regulator (WWTR1 or TAZ) in the Hippo pathway. One of the consequence of the pro-inflammatory environment provoked by exposure to asbestos fibers is the enhanced formation in the nucleus of the YAP/TAZ complex, which in turn promotes the expression of multiple proto-oncogenes, supporting cancer cell survival (42).

\section{Inherited BAP1 mutations and mesothelioma}

Epidemiological studies have provided evidence that only about $5 \%$ of miners and shipyard or manufacturing workers with prolonged exposure to asbestos developed mesothelioma (2). Studies on a mesothelioma epidemics among villagers in Cappadocia, Turkey, heavily exposed to erionite fibers and with an unusually high incidence of mesothelioma discovered the transmission of the susceptibility to mesothelioma in Mendelian autosomal dominant inheritance $(5,43)$.

The model of GxE interactions predicts that carriers of germline mutations have greater susceptibility to fiber-induced carcinogenesis and to the development of mesothelioma. This prompted the search of the gene(s) possibly involved. In two unrelated US families with no occupational exposure to asbestos and with high incidence of mesothelioma, array-comparative genomic hybridization (aCGH) and linkage analysis allowed the identification of possible frequent alterations at chromosome $3 \mathrm{p} 21$. Subsequent sequencing identified germline BAP1 mutations associated with autosomal dominant transmission of mesothelioma and uveal melanoma (44). In a parallel paper, germline mutations of $B A P 1$ were linked to dominant inheritance of melanocytic tumors (45). The individuals with germline mutated $B A P 1$ were also susceptible to other types of cancer like renal cell carcinoma and squamous cell carcinoma, leading to the identification of the BAP1 cancer syndrome (46).

Animal studies performed using $B a p 1^{+/}$heterozygous mice demonstrated that animals developed mesothelioma when exposed to ten-time lower doses of asbestos fibers that barely caused any mesothelioma in wild type Bap1 mice (47).

BAP1 was initially characterized as a nuclear protein with deubiquitylase activity, which is part of multiprotein transcriptional regulators of genes involved in metabolism, mitochondrial function, and cell proliferation (48). Nuclear BAP1 is also associated in chromatin remodeling (49), DNA double-strand repair $(50,51)$, and auto-deubiquitylation promoting its own nuclear localization (52). Recent studies demonstrated that BAP1 is endowed with a dual activity, both in the nucleus and in the cytoplasm, which cooperate to cause tumor suppression. Cytoplasmic BAP1 is prevalently localized in the endoplasmic reticulum (ER) fraction, where it deubiquitylates and stabilizes the type 3 inositol-1,4,5trisphosphate receptor (IP3R3). The function of IP3R3 is the release of $\mathrm{Ca}^{2+}$ from ER into the mitochondrial space through voltage-dependent anion channels (VDACs) of the outer membrane and the mitochondrial uniporter channel (MUC) of the inner mitochondrial membrane. The increase of $\mathrm{Ca}^{2+}$ concentration in the mitochondria induces the release of cytochrome c activating apoptosis. In heterozygous $B A P 1^{+-}$conditions, as in the individuals of the families with the BAP1 cancer syndrome, the reduced BAP1 dosage impairs both the DNA repair, accumulating DNA damage, and the apoptotic response. This dual effect positively selects cells carrying oncogenic mutations and promotes tumorigenesis (53). Moreover, the reduced BAP1 dosage in the cytoplasm has another consequence that was ascertained by metabolomics analysis. In non-tumoral cells, reduced BAP1 levels induced the Warburg effect, a shift of cell metabolism from oxidative phosphorylation (Krebs cycle) to aerobic glycolysis, originally identified as typical of cancer cells. However, the increase of aerobic glycolysis and lactate production in normal fibroblasts carrying heterozygous $B A P 1^{+/-}$indicates a new role for the Warburg effect in anticipating cancer onset by accelerating tumorigenesis (54).

Germline BAP1 mutations were associated with the increased risk of other tumors, like clear cell renal cell carcinoma $(55,56)$, cutaneous melanoma, ocular melanoma (57), atypical Spitz tumors $(46,58)$, basal cell carcinoma $(59,60)$, peritoneal mesothelioma (61-63), cholangiocarcinoma (64), and meningioma $(65,66)$. A worldwide genome analysis of $B A P 1$ germline mutations combined with the survey of the clinical features of the BAP1 cancer syndrome, showed the presence of the core syndrome tumors in a significant fraction of the families investigated by the study (67-69).

Further studies performed a genotype analysis in cancer patients, including those at high risk for familial inheritance, to identify germline alterations in additional genes possibly involved in the predisposition to mesothelioma and other cancers associated with $\mathrm{GxE}$ interactions $(62,70,71)$.

\section{Conclusions}

The cause of mesothelioma was uniquely attributed to asbestos exposure for a long time, however not all 
individuals exposed to asbestos and the other unregulated, naturally occurring carcinogenic mineral fibers like erionite, develop mesothelioma. The picture was further clarified when it was demonstrated that the genetic factors play critical roles in susceptibility to mesothelioma $(5,43)$. A progress in the knowledge on the causes of mesothelioma and other cancers related to the model of GxE interaction was the identification of $B A P 1$ as a predisposition gene for the development of familial mesothelioma (44), leading to the discovery of the BAP1 cancer syndrome $(46,48)$.

The mechanisms of cellular transformation following the exposure of HM to carcinogenic mineral fibers was elucidated by the discovery of the role of chronic inflammation mediated by HMGB1 and the inflammasome $(21,23)$. Moreover, the identification of BAP1 as a main controller of cell death and metabolism contributed to the definition of the complex array of molecular events mediated by asbestos carcinogenesis $(53,54)$.

Further studies will be required to identify the complete picture of the genes predisposing to mesothelioma and their contribution to the molecular mechanisms of asbestos carcinogenesis discovered so far, including chronic inflammation and altered metabolism.

\section{Acknowledgments}

Funding: This work was supported by Department of Defense Grant No. CA150671 to H Yang. The Riviera United 4-a Cure grant (H Yang).

\section{Footnote}

Conflicts of Interest: The authors have no conflicts of interest to declare.

Ethical Statement: The authors are accountable for all aspects of the work in ensuring that questions related to the accuracy or integrity of any part of the work are appropriately investigated and resolved.

Open Access Statement: This is an Open Access article distributed in accordance with the Creative Commons Attribution-NonCommercial-NoDerivs 4.0 International License (CC BY-NC-ND 4.0), which permits the noncommercial replication and distribution of the article with the strict proviso that no changes or edits are made and the original work is properly cited (including links to both the formal publication through the relevant DOI and the license). See: https://creativecommons.org/licenses/by-nc$\mathrm{nd} / 4.0 /$.

\section{References}

1. Carbone M, Yang H. Mesothelioma: recent highlights. Ann Transl Med 2017;5:238.

2. Carbone M, Adusumilli PS, Alexander HRJ, et al. Mesothelioma: Scientific Clues for Prevention, Diagnosis, and Therapy. CA Cancer J Clin 2019;69:402-29.

3. Baumann F, Ambrosi JP, Carbone M. Asbestos is not just asbestos: an unrecognised health hazard. Lancet Oncol 2013;14:576-8.

4. Carbone M, Baris YI, Bertino P, et al. Erionite exposure in North Dakota and Turkish villages with mesothelioma. Proc Natl Acad Sci U S A 2011;108:13618-23.

5. Carbone M, Emri S, Dogan AU, et al. A mesothelioma epidemic in Cappadocia: scientific developments and unexpected social outcomes. Nat Rev Cancer 2007;7:147-54.

6. Stanton MF, Laynard M, Tegeris A, et al. Carcinogenicity of fibrous glass: pleural response in the rat in relation to fiber dimension. J Natl Cancer Inst 1977;58:587-603.

7. Huang SX, Jaurand MC, Kamp DW, et al. Role of mutagenicity in asbestos fiber-induced carcinogenicity and other diseases. J Toxicol Environ Health B Crit Rev 2011;14:179-245.

8. Mossman BT. In vitro studies on the biologic effects of fibers: correlation with in vivo bioassays. Environ Health Perspect 1990;88:319-22.

9. Barlow CA, Grespin M, Best EA. Asbestos fiber length and its relation to disease risk. Inhal Toxicol 2017;29:541-54.

10. Carbone M, Yang H. Molecular pathways: targeting mechanisms of asbestos and erionite carcinogenesis in mesothelioma. Clin Cancer Res 2012;18:598-604.

11. Boulanger G, Andujar P, Pairon JC, et al. Quantification of short and long asbestos fibers to assess asbestos exposure: a review of fiber size toxicity. Environ Health 2014;13:59.

12. Bernstein DM, Donaldson K, Decker U, et al. A biopersistence study following exposure to chrysotile asbestos alone or in combination with fine particles. Inhal Toxicol 2008;20:1009-28.

13. Qi F, Okimoto G, Jube S, et al. Continuous exposure to chrysotile asbestos can cause transformation of human mesothelial cells via HMGB1 and TNF-alpha signaling. Am J Pathol 2013;183:1654-66. 
14. Larson D, Powers A, Ambrosi JP, et al. Investigating palygorskite's role in the development of mesothelioma in southern Nevada: Insights into fiber-induced carcinogenicity. J Toxicol Environ Health B Crit Rev 2016;19:213-30.

15. Nagai H, Ishihara T, Lee WH, et al. Asbestos surface provides a niche for oxidative modification. Cancer Sci 2011;102:2118-25.

16. $\mathrm{Xu} \mathrm{A}, \mathrm{Wu}$ LJ, Santella RM, et al. Role of oxyradicals in mutagenicity and DNA damage induced by crocidolite asbestos in mammalian cells. Cancer Res 1999;59:5922-6.

17. Toyokuni S. Iron addiction with ferroptosis-resistance in asbestos-induced mesothelial carcinogenesis: Toward the era of mesothelioma prevention. Free Radic Biol Med 2019;133:206-15.

18. Ramos-Nino ME, Blumen SR, Sabo-Attwood T, et al. HGF mediates cell proliferation of human mesothelioma cells through a PI3K/MEK5/Fra-1 pathway. Am J Respir Cell Mol Biol 2008;38:209-17.

19. Broaddus VC, Yang L, Scavo LM, et al. Asbestos induces apoptosis of human and rabbit pleural mesothelial cells via reactive oxygen species. J Clin Invest 1996;98:2050-9.

20. Yang H, Bocchetta M, Kroczynska B, et al. TNFalpha inhibits asbestos-induced cytotoxicity via a NFkappaB-dependent pathway, a possible mechanism for asbestos-induced oncogenesis. Proc Natl Acad Sci U S A 2006;103:10397-402.

21. Yang H, Rivera Z, Jube S, et al. Programmed necrosis induced by asbestos in human mesothelial cells causes high-mobility group box 1 protein release and resultant inflammation. Proc Natl Acad Sci U S A 2010;107:12611-6.

22. Kadariya Y, Menges CW, Talarchek J, et al. InflammationRelated IL-1beta/IL-1R Signaling Promotes the Development of Asbestos-Induced Malignant Mesothelioma. Cancer Prev Res (Phila) 2016;9:406-14.

23. Thompson JK, Shukla A, Leggett AL, et al. Extracellular signal regulated kinase 5 and inflammasome in progression of mesothelioma. Oncotarget 2018;9:293-305.

24. Yang H, Pellegrini L, Napolitano A, et al. Aspirin delays mesothelioma growth by inhibiting HMGB1-mediated tumor progression. Cell Death Dis 2015;6:e1786.

25. Pellegrini L, Xue J, Larson D, et al. HMGB1 targeting by ethyl pyruvate suppresses malignant phenotype of human mesothelioma. Oncotarget 2017;8:22649-61.

26. Jube S, Rivera ZS, Bianchi ME, et al. Cancer cell secretion of the DAMP protein HMGB1 supports progression in malignant mesothelioma. Cancer Res 2012;72:3290-301.

27. Bonaldi T, Talamo F, Scaffidi P, et al. Monocytic cells hyperacetylate chromatin protein HMGB1 to redirect it towards secretion. EMBO J 2003;22:5551-60.

28. Evankovich J, Cho SW, Zhang R, et al. High mobility group box 1 release from hepatocytes during ischemia and reperfusion injury is mediated by decreased histone deacetylase activity. J Biol Chem 2010;285:39888-97.

29. Yang Z, Li L, Chen L, et al. PARP-1 mediates LPSinduced HMGB1 release by macrophages through regulation of HMGB1 acetylation. J Immunol 2014;193:6114-23.

30. Napolitano A, Antoine DJ, Pellegrini L, et al. HMGB1 and Its Hyperacetylated Isoform are Sensitive and Specific Serum Biomarkers to Detect Asbestos Exposure and to Identify Mesothelioma Patients. Clin Cancer Res 2016;22:3087-96.

31. Affar EB, Carbone M. BAP1 regulates different mechanisms of cell death. Cell Death Dis 2018;9:1151.

32. Carbone M, Amelio I, Affar EB, et al. Consensus report of the 8 and 9th Weinman Symposia on Gene $\mathrm{x}$ Environment Interaction in carcinogenesis: novel opportunities for precision medicine. Cell Death Differ 2018;25:1885-904.

33. Ly P, Cleveland DW. Rebuilding Chromosomes After Catastrophe: Emerging Mechanisms of Chromothripsis. Trends Cell Biol 2017;27:917-30.

34. Yoshikawa Y, Emi M, Hashimoto-Tamaoki T, et al. Highdensity array-CGH with targeted NGS unmask multiple noncontiguous minute deletions on chromosome 3 p21 in mesothelioma. Proc Natl Acad Sci U S A 2016;113:13432-7.

35. Oey H, Daniels M, Relan V, et al. Whole-genome sequencing of human malignant mesothelioma tumours and cell lines. Carcinogenesis 2019;40:724-34.

36. Mansfield AS, Peikert T, Smadbeck JB, et al. Neoantigenic Potential of Complex Chromosomal Rearrangements in Mesothelioma. J Thorac Oncol 2019;14:276-87.

37. Husain AN, Colby TV, Ordonez NG, et al. Guidelines for Pathologic Diagnosis of Malignant Mesothelioma 2017 Update of the Consensus Statement From the International Mesothelioma Interest Group. Arch Pathol Lab Med 2018;142:89-108.

38. Jongsma J, van Montfort E, Vooijs M, et al. A conditional mouse model for malignant mesothelioma. Cancer Cell 2008;13:261-71.

39. Altomare DA, Menges CW, Pei J, et al. Activated TNF- 
alpha/NF-kappaB signaling via down-regulation of Fasassociated factor 1 in asbestos-induced mesotheliomas from Arf knockout mice. Proc Natl Acad Sci U S A 2009;106:3420-5.

40. Sato T, Sekido Y. NF2/Merlin Inactivation and Potential Therapeutic Targets in Mesothelioma. Int J Mol Sci 2018;19. doi: 10.3390/ijms19040988.

41. Altomare DA, Vaslet CA, Skele KL, et al. A mouse model recapitulating molecular features of human mesothelioma. Cancer Res 2005;65:8090-5.

42. Rehrauer H, Wu L, Blum W, et al. How asbestos drives the tissue towards tumors: YAP activation, macrophage and mesothelial precursor recruitment, RNA editing, and somatic mutations. Oncogene 2018;37:2645-59.

43. Roushdy-Hammady I, Siegel J, Emri S, et al. Geneticsusceptibility factor and malignant mesothelioma in the Cappadocian region of Turkey. Lancet 2001;357:444-5.

44. Testa JR, Cheung M, Pei J, et al. Germline BAP1 mutations predispose to malignant mesothelioma. Nat Genet 2011;43:1022-5.

45. Wiesner T, Obenauf AC, Murali R, et al. Germline mutations in BAP1 predispose to melanocytic tumors. Nat Genet 2011;43:1018-21.

46. Carbone M, Ferris LK, Baumann F, et al. BAP1 cancer syndrome: malignant mesothelioma, uveal and cutaneous melanoma, and MBAITs. J Transl Med 2012;10:179.

47. Napolitano A, Pellegrini L, Dey A, et al. Minimal asbestos exposure in germline BAP1 heterozygous mice is associated with deregulated inflammatory response and increased risk of mesothelioma. Oncogene 2016;35:1996-2002.

48. Carbone M, Yang H, Pass HI, et al. BAP1 and cancer. Nat Rev Cancer 2013;13:153-9.

49. Lee HS, Lee SA, Hur SK, et al. Stabilization and targeting of INO80 to replication forks by BAP1 during normal DNA synthesis. Nat Commun 2014;5:5128.

50. Ismail IH, Davidson R, Gagne JP, et al. Germline mutations in BAP1 impair its function in DNA doublestrand break repair. Cancer Res 2014;74:4282-94.

51. Yu H, Pak H, Hammond-Martel I, et al. Tumor suppressor and deubiquitinase BAP1 promotes DNA double-strand break repair. Proc Natl Acad Sci U S A 2014;111:285-90.

52. Mashtalir N, Daou S, Barbour H, et al.

Autodeubiquitination protects the tumor suppressor BAP1 from cytoplasmic sequestration mediated by the atypical ubiquitin ligase UBE2O. Mol Cell 2014;54:392-406.

53. Bononi A, Giorgi C, Patergnani S, et al. BAP1 regulates IP3R3-mediated $\mathrm{Ca}(2+)$ flux to mitochondria suppressing cell transformation. Nature 2017;546:549-53.

54. Bononi A, Yang H, Giorgi C, et al. Germline BAP1 mutations induce a Warburg effect. Cell Death Differ 2017;24:1694-704.

55. Pena-Llopis S, Vega-Rubin-de-Celis S, Liao A, et al. BAP1 loss defines a new class of renal cell carcinoma. Nat Genet 2012;44:751-9.

56. Popova T, Hebert L, Jacquemin V, et al. Germline BAP1 mutations predispose to renal cell carcinomas. Am J Hum Genet 2013;92:974-80.

57. Njauw CN, Kim I, Piris A, et al. Germline BAP1 inactivation is preferentially associated with metastatic ocular melanoma and cutaneous-ocular melanoma families. PLoS One 2012;7:e35295.

58. Murali R, Wilmott JS, Jakrot V, et al. BAP1 expression in cutaneous melanoma: a pilot study. Pathology 2013;45:606-9.

59. de la Fouchardiere A, Cabaret O, Savin L, et al. Germline BAP1 mutations predispose also to multiple basal cell carcinomas. Clin Genet 2015;88:273-7.

60. Wadt KA, Aoude LG, Johansson P, et al. A recurrent germline BAP1 mutation and extension of the BAP1 tumor predisposition spectrum to include basal cell carcinoma. Clin Genet 2015;88:267-72.

61. Kittaneh M, Berkelhammer C. Detecting germline BAP1 mutations in patients with peritoneal mesothelioma: benefits to patient and family members. J Transl Med 2018;16:194.

62. Panou V, Gadiraju M, Wolin A, et al. Frequency of Germline Mutations in Cancer Susceptibility Genes in Malignant Mesothelioma. J Clin Oncol 2018;36:2863-71.

63. Rai K, Pilarski R, Cebulla CM, et al. Comprehensive review of BAP1 tumor predisposition syndrome with report of two new cases. Clin Genet 2016;89:285-94.

64. Pilarski R, Cebulla CM, Massengill JB, et al. Expanding the clinical phenotype of hereditary BAP1 cancer predisposition syndrome, reporting three new cases. Genes Chromosomes Cancer 2014;53:177-82.

65. Abdel-Rahman MH, Pilarski R, Cebulla CM, et al. Germline BAP1 mutation predisposes to uveal melanoma, lung adenocarcinoma, meningioma, and other cancers. J Med Genet 2011;48:856-9.

66. Shankar GM, Abedalthagafi M, Vaubel RA, et al. Germline and somatic BAP1 mutations in high-grade rhabdoid meningiomas. Neuro Oncol 2017;19:535-45.

67. Walpole S, Pritchard AL, Cebulla CM, et al. Comprehensive Study of the Clinical Phenotype of 
Germline BAP1 Variant-Carrying Families Worldwide. J Natl Cancer Inst 2018;110:1328-41.

68. Baumann F, Flores E, Napolitano A, et al. Mesothelioma patients with germline BAP1 mutations have 7-fold improved long-term survival. Carcinogenesis 2015;36:76-81.

69. Carbone M, Flores EG, Emi M, et al. Combined Genetic and Genealogic Studies Uncover a Large BAP1 Cancer Syndrome Kindred Tracing Back Nine Generations to a Common Ancestor from the 1700s. PLoS Genet

Cite this article as: Gaudino G, Xue J, Yang H. How asbestos and other fibers cause mesothelioma. Transl Lung Cancer Res 2020;9(Suppl 1):S39-S46. doi: 10.21037/tlcr.2020.02.01 2015;11:e1005633.

70. Pastorino S, Yoshikawa Y, Pass HI, et al. A Subset of Mesotheliomas With Improved Survival Occurring in Carriers of BAP1 and Other Germline Mutations. J Clin Oncol 2018:JCO2018790352. [Epub ahead of print].

71. Yoshikawa Y, Sato A, Tsujimura T, et al. Biallelic germline and somatic mutations in malignant mesothelioma: multiple mutations in transcription regulators including mSWI/SNF genes. Int J Cancer 2015;136:560-71. 